\title{
Active Galactic Nuclei: Jets as the Source of Hadrons and Neutrinos
}

\author{
Athina Meli ${ }^{1,2,3}$, Paolo Ciarcelluti ${ }^{3}$ \\ ${ }^{1}$ Department of Physics and Astronomy, University of Gent, Belgium \\ ${ }^{2}$ IFPA, Department of Astrophysics, Geophysics and Oceanography, University of Liege, Belgium \\ ${ }^{3}$ Web Institute of Physics, www.wiph.org
}

Corresponding author: athina.meli@ugent.be

\begin{abstract}
Active galactic nuclei are extragalactic sources, and their relativistic hot-plasma jets are believed to be the main candidates of the cosmic-ray origin, above the so-called knee region of the cosmic-ray spectrum. Relativistic shocks, either single or multiple, have been observed or been theorized to be forming within relativistic jet channels in almost all active galactic nuclei sources. The acceleration of non-thermal particles (e.g. electrons, protons) via the shock Fermi acceleration mechanism, is believed to be mainly responsible for the power-law energy distribution of the observed cosmic-rays, which in very high energies can consequently radiate high energy gamma-rays and neutrinos, through related radiation channels. Here, we will focus on the primary particle (hadronic) shock acceleration mechanism, and we will present a comparative simulation study of the properties of single and multiple relativistic shocks, which occur in AGN jets. We will show that the role of relativistic (quasi-parallel either quasi-perpendicular) shocks, is quite important since it can dramatically alter the primary CR spectral indices and acceleration efficiencies. These properties being carried onto gamma-ray and neutrino radiation characteristics, makes the combination of them a quite appealing theme for relativistic plasma and shock acceleration physics, as well as observational cosmic-ray, gamma-ray and neutrino astronomy.
\end{abstract}

Keywords: active galactic nuclei - jets - relativistic shocks - cosmic rays - acceleration - gamma-rays - neutrinos.

\section{Introduction}

Cosmic Rays (CRs) are subatomic particles (e.g. protons) and radiation of extra-terrestrial origin. Their energies range between a few $\mathrm{eV}$ to hundreds of $\mathrm{PeV}$. These particles register a power-law spectrum, and the low-energy ones are believed to originate from galactic, while the CRs with higher energies originate from extra-galactic sources. Specifically, CRs above energies of $\sim 10^{17} \mathrm{eV}$, almost certainly originate from extragalactic sources such as Active Galactic Nuclei (AGN) and their relativistic jets.

It is the particle shock acceleration in the superalfvenic jet plasmas, which is believed to be the main mechanism responsible for the production of the nonthermal CRs (e.g. Krymskii, 1977). Especially, the importance of the very high energy $\mathrm{CR}$ acceleration is a favourable theme of study, as it is also the TeV photons and neutrinos theme, which are produced either by the primary proton-photon or primary proton-nucleon interactions, offering further insights about the environment of a considered astrophysical source.

AGN sources are actually a compact region at the centre of a galaxy, which is believed to be a supermassive black hole. The radiation from an AGN is believed to be mainly a result by the accretion of mass around the black hole. The accretion of matter around the spinning core of an AGN produces twin, highly collimated fast outflows of hot gas, called jets, extending to distances of a few parsecs to thousands of parsecs. It is of importance to mention that the physics of jet mechanism and the jet composition on very small scales, are not perfectly understood at present. We know thought, that the AGN black holes and their jets radiate in all wavebands, from the radio up to the gamma-ray regime via mostly the synchrotron and the inverse-Compton scattering processes. Moreover with a typical AGN luminosity of $L \sim 10^{42}-10^{47} \mathrm{erg} / \mathrm{s}$, it is possible to explain the origin of the very high energy CR flux, under the assumption that these objects follow the star-formation-rate. For example, Moran et al. (2001) find an average luminosity from an estimated 95 X-ray active AGN within $60 \mathrm{Mpc}$ of $4.8 \times 10^{44} \mathrm{erg} / \mathrm{s}$, this distance being the $\mathrm{CR}$ absorption horizon at about $2 \times 10^{20} \mathrm{eV}$. Within the regions of local space accessible to $\mathrm{CR}$ diffusion, the energy supply over a Hubble time is $6.6 \times 10^{-16} \mathrm{erg} / \mathrm{cm}^{3}$. By comparison, the Gamma Ray Bursts supply is $6.7 \times 10^{-20} \mathrm{erg} / \mathrm{cm}^{3}$. Thus, AGN as the most luminous permanent sources in the sky can stand as the most likely candidate for the acceleration of very 
high energy CRs. Interpretation of data on the electron synchrotron radiation observed in the radio regime, suggests the presence of mildly-relativistic shocks with mean boost factors of $\Gamma=10-30$ in the jets of AGN, see Biermann and Strittmatter (1987). Moreover, assuming that hadrons (as we do in this work) are also accelerated along with the electrons in the jet, justify AGN to be as good candidates as to provide at least a significant fraction of the extragalactic component of the hadronic CR flux observed.

Here we will discuss the primary particle shock acceleration mechanism in AGN jets, in the context of comparative test-particle simulation studies, for relativistic single and multiple quasi-parallel and quasiperpendicular shocks. We will also briefly highlight the possible consequent TeV-photon and neutrino emission.

\section{Single-Relativistic Shocks}

Here we will review shock acceleration simulations, based on the relativistic single-shock, test-particle Monte Carlo code of Meli and Quenby (2003a,b), as a comparative tool to the multiple-shock acceleration study of the following section. In our simulations we inject a large number of particles $N=10^{4}$ upstream towards a single planar shock, with an initial particle energy $\gamma=\Gamma_{s h}+5$. We use shock Lorentz factors of $\Gamma=10$ and 30 , and we numerically let the particles' guiding-centers to scatter with the assumed background magnetic field irregularities, around a shock region of a length $d=2 \mathrm{pc}$. For details on the code see Meli and Quenby (2003a,b) and Meli et al. (2008).

Briefly, our exemplary simulations show that : the relativistic quasi-parallel (e.g. $\psi=25^{\circ}$ ) and quasiperpendicular (e.g. $\quad \psi=75^{\circ}$ ) single-shock acceleration mechanism manifests into a clear deviation of the CR spectral index (compared to standard, unaffected non-relativistic spectral index of $\sim 2.0$ ) with an absent 'universal' power-law spectrum, which depends on three parameters: i) speed of the shock, ii) shock inclination, and iii) different scattering modes, see Table 1. We clearly see a high acceleration efficiency (in terms of $E_{\max }$ ) for the cases of quasi-parallel shocks. Albeit, quasi-perpendicular shocks result into more 'conservative' acceleration efficiencies and lower attained energies.

It is important to note that it is been reported that the lower the turbulence, in terms of the mean-free-path $\eta=\lambda / r_{g}$, the steeper the particle spectrum (Meli et al. 2008). Near-perpendicular shocks strongly appear to give the steepest spectra compared to quasi-parallel shock cases, and are the less efficient accelerators, see also e.g. Niemec and Ostrowski (2004), Stecker et al. (2007), etc.

Summarizing, it is widely accepted that (with a few exceptions) as soon as an astrophysical shock speed becomes comparable to the speed of light, the power-law index of the accelerated CR spectra, depends strongly not only on the speed of the shock but also on the shock obliquity (scattering mode is also important, see Meli, 2011), identifying clearly flatter spectra for small shock inclinations, with greater acceleration efficiencies.

Table 1: Examples of simulations for single shocks with different shock Lorentz factors $(\Gamma)$ and shock inclination angles $(\psi)$, and the attained spectral indices $(\sigma)$, and maximal particle energies $E$, respectively.

\begin{tabular}{|l|l|l|}
\hline \hline$\Gamma$ & $\psi=25^{o}$ & $\psi=75^{\circ}$ \\
\hline \hline 10 & $\sigma=2.0, E=10^{9.3} \mathrm{GeV}$ & $\sigma=2.4, E=10^{5.6} \mathrm{GeV}$ \\
\hline 30 & $\sigma=1.9, E=10^{9.8} \mathrm{GeV}$ & $\sigma=2.3, E=10^{6.0} \mathrm{GeV}$ \\
\hline
\end{tabular}

\section{Multiple-Relativistic Shocks}

After the brief insights on the relativistic single-shock acceleration above, we will now discuss a case study of particle acceleration in multiple-shocks, based on the fully relativistic multiple-shock, test-particle Monte Carlo code by Meli and Biermann (2013). In the past it was shown that for an infinite number of subsequent non-relativistic shocks with injection at each shock, the flattening of the spectrum (compared to a single shock) extends even to the highest energy particles with a momentum dependence of $f(p) \propto p^{-3}$ (e.g. White 1985). Here we will investigate the acceleration mechanism in relativistic, multiple, quasi-parallel and quasiperpendicular shocks. We can envisage blobs of hot plasma ejected by the black hole of an AGN that can travel along the jet and pass through a sequence of shocks, as it has been also observed in BLac PKS 1510089 (Marscher et al. 2010) and other sources.

Here we numerically calculate the scattering of the particles' guiding-center with pre-determined background magnetic field irregularities, around multipleshock regions and within a distance $d=5 \mathrm{pc}$. At the beginning of the acceleration we allow a single initial injection of particles of a fixed number $N=10^{4}$, with an initial $\gamma=\Gamma_{s h}+5$, and shock Lorentz factor $\Gamma=30$ (for the first two shocks) and $\Gamma=10$ (for the second pair), justified by the decompression-compression conditions of the downstream plasma (Melrose and Pope 1993) of the precedent shock pattern (i.e., a velocity compression ratio $r=3$ ). That is, during the acceleration we allow a probability of escape $P_{e s c}=0.1$ between shocks due to decompression effects. The escape probability gives the fraction of particles of the downstream distribution of each shock, that will not be accelerated 
more. These particles remain in the system and contribute directly to the downstream distribution of the 4 -th shock. This factor would naturally result in steeper spectral slopes comparing to a single shock acceleration spectrum as the relativistic conditions render the spectra flatter. Assuming also a field compression ratio $r_{B_{c}}$ equal to , and an assumed magnetic field strength of the order of a few mgauss, the distance between each shock is safely set as $d=1 \mathrm{pc}$.

We study two cases of shock patterns: i) a set four quasi-parallel shocks (with the same apex to the assumed jet axis), and inclination angles $\psi_{1}, \psi_{2}, \psi_{3}, \psi_{4}=$ $25^{\circ}$, and ii) a set of four quasi-perpendicular shocks and inclination angles $\psi_{1}, \psi_{2}, \psi_{3}, \psi_{4}=75^{\circ}$.

\section{Table 2:}

\begin{tabular}{|l|l|l|l|}
\hline \hline$\Gamma$ & $\psi$ & $\sigma$ & $E[\mathrm{GeV}]$ \\
\hline \hline 30 & $25^{\circ}$ & 2.6 & $10^{5.9}$ \\
\hline 30 & $25^{\circ}$ & 2.2 & $10^{6.9}$ \\
\hline 10 & $25^{\circ}$ & 2.1 & $10^{8.6}$ \\
\hline 10 & $25^{\circ}$ & 1.5 & $10^{9.5}$ \\
\hline \hline
\end{tabular}

Table 3: In the tables above we see indicative simulation numbers for two sets of four-shocks, their maximal attained energies $E$ at each shock downstream, and their spectral indices $\sigma$. Shock inclination angles are denoted as $\psi$, and $\Gamma$ is the shock Lorentz factor.

\begin{tabular}{|l|l|l|l|}
\hline \hline$\Gamma$ & $\psi$ & $\sigma$ & $E[\mathrm{GeV}]$ \\
\hline \hline 30 & $75^{\circ}$ & 2.7 & $10^{4.6}$ \\
\hline 30 & $75^{\circ}$ & 2.4 & $10^{5.2}$ \\
\hline 10 & $75^{\circ}$ & 2.3 & $10^{5.9}$ \\
\hline 10 & $75^{\circ}$ & 2.2 & $10^{6.4}$ \\
\hline \hline
\end{tabular}

Briefly, from the tables above, one notices that (i) the spectra by each shock in every shock-set become gradually flatter, following on one hand the findings of single relativistic shocks discussed in the previous section, and on the other hand the trends reported in e.g. Melrose and Pope (1993) and Gieseler and Jones (2000) for multiple shocks. (ii) The flatness of the spectra is more evident for the consecutive quasi-parallel shocks than for the quasi-perpendicular ones, following, as expected, the individual shock effects we discussed in the previous paragraph. (iii) The acceleration in the case of quasi-parallel shocks seems very efficient and comparable to single shock acceleration.

\section{Neutrino and Gamma-Ray Astronomy}

Over the years many models have been created (e.g. Stecker, 2005), assuming protons are accelerated at the shocks of AGN jets and interact with different photon fields to produce high energy gamma-rays and neutrinos. The $\mathrm{CR}$ acceleration cases shown here, have implications for neutrino and gamma-ray astronomy, and especially for the $\mathrm{TeV}$ sources. We know that only from e.g. the $p-\gamma$ interaction (where the optical depth must be equal or larger than 1), the consequent decay of the $\pi^{0}$ results into a high energy photon (in 0.33 of the cases). Also $\pi^{ \pm}$particles produce neutrinos. Specifically, the probability for $\pi^{+}$production is 0.66 . Around 0.20 of the proton energy goes into pion, and the products carry equal energy, so that a fraction of $0.25(0.50)$ is transferred to each neutrino (photon) and furthermore, one must also consider that neutrinos oscillate. Therefore the high-energy photon and neutrino spectra can be connected as $\frac{d N_{\nu}}{d E_{\nu}}=\frac{1}{8} \cdot \frac{d N_{\gamma}}{d E_{\gamma}}$. Additionally it can be assumed that the neutrino carries about $1 / 20$ th of the proton's energy (see Halzen, 2008). All these can be very helpful in understanding CRs and related consequent emissions. Nevertheless, in many AGN the photon signal at $\mathrm{TeV}$ energies is not unique, since leptonic processes like Inverse Compton scattering could also contribute at the same energies. But there is a way of identifying hadronic interactions in astrophysical shocks by estimating the flux of neutrinos. Not to let unmentioned, one can also assume that the spectral indices for the $\mathrm{TeV}$ photon and neutrino emission at a break threshold energy, generally at around $10^{2} \mathrm{GeV}$, can be considered to be about the same.

It is accepted that not all AGN sources can be seen or inferred emitting CRs, and this due to either the high galactic background absorptions, or particle's random diffusion in magnetic fields, or even insufficient power to accelerate very high CRs. Nevertheless, neutrino signals, could reveal the possible hadronic nature of a $\mathrm{TeV}$ source.

We saw in this work that relativistic quasiperpendicular shocks in AGN, single or multiple, cannot accelerate CRs to very high energies. We know also that a photon field in the jet of an AGN can range from low energies to many TeV. We also know (e.g. Becker et al. 2011) that the proton energy necessary to produce a Delta resonance in the observer's frame, can be given as, $E_{p} \geq \frac{\Gamma^{2}}{(1+z)^{2}} \frac{m_{\Delta}^{2}-m_{p}^{2}}{4} \cdot E_{\gamma}^{-1}$, where $m_{\Delta}$ is the mass of the Delta resonance, $m_{p}$ is the proton-mass, $E_{\gamma}$ is the characteristic photon energy and $z$ is the redshift of the source (e.g. Cen A, $z=0.001$ ). Therefore, simplistically, one can assume a photon field (i.e. but dense enough for photo-hadronic interactions) to make 
an estimation of the maximal energy of the proton (as shown in this work) necessary to produce TeV-photons and $\mathrm{GeV}-\mathrm{TeV}$ neutrinos, or vice-versa, and make further estimations on the correlations of different wavelengths from the same source.

As an example here for the case of the favourable $\mathrm{TeV}$ emitter, Cen A, with $z=0.001$ and an assumed $\operatorname{shock}(\mathrm{s})$ Lorentz factor of $\Gamma=30$, the proton energy sufficient to produce a Delta resonance and with these, $\mathrm{TeV}$-photos and neutrinos, can be given as

$$
E_{p} \geq 10^{5} \cdot(1+z)^{-2} \cdot\left(\frac{\Gamma}{30}\right)^{2} \cdot\left(\frac{E_{\gamma}}{1 \mathrm{MeV}}\right)^{-1} \mathrm{GeV} .
$$

Thus, for quasi-perpendicular shocks, the proton spectra found in our simulations reach low energies $E_{p} \sim$ $10^{6} \mathrm{GeV}$, which could not be directly observed. In order though to achieve $\mathrm{TeV}$ photons and along with them neutrinos, one would require photon field energies at the jet of equal or greater than $E_{\gamma} \geq 10 \mathrm{MeV}$. Of course these energies can be produced via the Inverse Compton scattering of synchrotron or external photons with the accelerated electrons certainly present at the jet. Therefore one would expect a high energy signal of neutrinos, if the source is of a hadronic nature.

What one can in generally conclude from the above brief discussion, is that the extragalactic $\mathrm{TeV}$ sources, which can not be observed as candidates of ultra-high energy CRs, may nevertheless emit high energy neutrinos implying the hadronic nature of the cosmic rays. This fact can be used as a supplementary tool to filterout neutrino source candidates. Calculations on flux and further details on radiative consequences are under away.

\section{Conclusions}

The relativistic shocks in the jets of AGN are a favourable accelerator of high-energy as well as lowenergy CRs, via the shock acceleration mechanism. Here, we performed comparative Monte-Carlo simulation studies for single and multiple relativistic shocks, assuming protons as the primary accelerated particle and we showed that relativistic (quasi-parallel either quasi-perpendicular) shocks are very important since they can alter dramatically the CR spectral indices and acceleration efficiency. These properties are carried onto gamma-ray and neutrino emission from the same sources. Thus, assuming a hadronic primary accelerated scenario, one could have an additional understanding regarding expected neutrino fluxes from $\mathrm{TeV}$ sources. Specifically, AGN with TeV gamma-ray emission, and non-observable or low-energy CRs, could have the potential to carry an additional high energy neutrino flux signal, mimicking accordingly the primary accelerated particle spectra in the jet, separating it from the Inverse Compton leptonic or other related processes. Accurate flux expectancies of gamma-rays and mostly neutrinos within Earth neutrino-observatory potentials (e.g. Icecube, KM3Net), is the next step for deeper understanding of AGN jets.

\section{Acknowledgement}

A.M. would like to thank the organisers for the invitation to give an advanced talk on the topic of shock acceleration and cosmic rays. She also likes to thank P. L. Biermann, J. J. Quenby and J. K. Becker for contributing their valuable expertise to the cited studies above; she acknowledges fruitful discussions with Antares and Icecube/Icetop collaborations; and she is constantly indebted to A. Mastichiadis, S. Dimitrakoudis, R. Protheroe, A. Donea, T. Kneiske, M. Dieckmann, A. Reimer, M. Ostrowski, I. Brancus, J. Wentz, M. Giller, A. Wolfendale, T. Stanev, K.Nishikawa for offering, in different occasions over the last years, valuable advice and insights on related studies.

\section{References}

[1] Becker, J., Meli, A. \& Biermann P. L., 2011 Nucl. Ist. Meth., 630269 doi:10.1016/j.nima.2010.06.082

[2] Biermann, P. L. \& Strittmatter, P. A., 1987 ApJ, 322, 643

[3] Gieseler, J. \& Jones, T. W., 2000, AA 357, 1133

[4] Halzen, F., J., 2008 Phys. Conf. Ser., 120 (6) 062004 doi:10.1088/1742-6596/120/6/062004

[5] Krymskii, G. F., 1977 Akademiia Nauk SSSR, 234, 1306

[6] Marscher, A. P., Jorstad, S. G., Larionov, V. M., et al., 2010 ApJ, 710L, 126 doi:10.1088/2041-8205/710/2/L126

[7] Meli, A. \& Quenby, J. J., 2003b APh 19, 649

[8] Meli, A., Becker, J. K., J., Quenby, J. J., 2008 A\&A 492,323

[9] Meli, A., 2011, AstrSpSc 7, 287

[10] Meli, A \& P. . Biermann, 2013 A\&A in press

[11] Melrose, D. B. \& Pope, M. H., 1993 PASAu, 10, 222

[12] Moran, E. C., Kay, L. E., Davis. M., Filippenko, A. V., Barth., 2001 Ast.J., 566, L75

[13] Niemiec, J. \& Ostrowski, M., 2004, ApJ 610, 851 doi:10.1086/421730

[14] Stecker F. W., 2005 PhRvD, 72j 7301

[15] Stecker F. W., Baring J., Summerlin E. J., 2007 ApJ, 667L, 29 doi:10.1086/522005

[16] White R. L., 1985 ApJ 289, 698

\section{DISCUSSION}

PIETER MEINTJES: You mentioned that strong relativistic shocks will be accompanied by turbulence. Can you comment on the role that MHD can possibly play in the 
acceleration process? Is it possible that strong MHD turbulence can distort a power-law spectrum into a kind of relativistic Maxwellian as a result of acceleration through MHD waves? If this happens, under what conditions?

ATHINA MELI: The numerical approach of the model presented here is that of the test-particle approach, investigating a collision-less plasma (kinetic theory) and not a collisional plasma (fluid theory). I note that shocks do exist in collisionless media, i.e. without collisional dissipation (resistivity/viscosity). Let me also add though that an MHD description of a collisionless plasma it is not entirely wrong to be assumed, because all fluid equations (except closure) are exact conservation laws (and closure is not too sensitive to microphysics at large scales), but there are many other exceptions mainly when large scales are not independent of the small ones with critical limits. For the present case, the MHD turbulence effect connection can be attained at the context of assuming the strength of scattering modes directly in the code (i.e. strong or weak scattering, as large angle diffusion or pitch angle scattering), by relating it to the diffusion coefficient $(\kappa)$ which is dependent on the level of media turbulence, denoted by the ratio $\lambda / r_{g}$. Please see detailed descriptions of the particle kinematics in Meli and Quenby (2003b), Meli et al.(2008) and Meli (2011). As I discussed here, the type of scattering hence the level of MHD turbulence in the media can alter the produced spectra in the relativistic shock acceleration cases.
JIM BEALL: Does the spectra you get from these shocks depend on the specific shock structure?

ATHINA MELI: The shock structure can alter the steepness of the shock, and I deal with this matter in another recent work (Meli, 2013) (not presented here, planar shocks only).

MAURIZION SPURLO: Cosmic rays with energies above $10^{19} \mathrm{eV}$, following the AUGER measurements, seem to be mainly heavy nuclei. Can the acceleration model from AGN jets you presented, accelerate heavy nuclei instead protons maintaining the same features?

ATHINA MELI: The relativistic shocks presented here produce spectra mainly below $10^{19} \mathrm{eV}$, favoured for protons. Nevertheless, in some ideal cases one can obtain easily spectra that reach energies of $10^{20-21} \mathrm{eV}$, as I showed in a related work. This acceleration model can treat the acceleration of heavy nuclei, but the consideration of added losses must be taken into account in the code, which will mostly steepen the spectrum by an order of magnitude or more, as some of my preliminary test-runs have shown. I note here though an important point: The spectra even above energies of $10^{19} \mathrm{eV}$ seem to be a (unknown) mix of protons and heavy nuclei, since pure heavy nuclei population seems highly improbable. 